\title{
Resting-state functional connectivity imaging of the mouse brain using photoacoustic tomography
}

Mohammadreza Nasiriavanaki, Jun Xia, Hanlin Wan, Adam Q. Bauer, Joseph P. Culver, et al.

Mohammadreza Nasiriavanaki, Jun Xia, Hanlin Wan, Adam Q. Bauer, Joseph P. Culver, Lihong V. Wang, "Resting-state functional connectivity imaging of the mouse brain using photoacoustic tomography," Proc. SPIE 8943, Photons Plus Ultrasound: Imaging and Sensing 2014, 894320 (3 March 2014); doi: 10.1117/12.2036606

SPIE. Event: SPIE BiOS, 2014, San Francisco, California, United States 


\title{
Resting-state functional connectivity imaging of the mouse brain using photoacoustic tomography
}

\author{
Mohammadreza Nasiriavanaki ${ }^{\text {a }}$, Jun Xia ${ }^{a}$, Hanlin Wan ${ }^{a}$, Adam Q. Bauer ${ }^{b}$, Joseph P. Culver ${ }^{b}$, and Lihong V. Wang ${ }^{a}$ \\ ${ }^{\text {a }}$ Optical Imaging Laboratory, Department of Biomedical Engineering, Washington University in St. Louis, St. Louis, MO 63130, USA \\ ${ }^{\mathrm{b}}$ Department of Radiology, Washington University in St. Louis, St. Louis, MO 63110, USA \\ Corresponding author. Email: LHWANG@WUSTL.EDU
}

\begin{abstract}
Resting-state functional connectivity (RSFC) imaging is an emerging neuroimaging approach that aims to identify spontaneous cerebral hemodynamic fluctuations and their associated functional connections. Clinical studies have demonstrated that RSFC is altered in brain disorders such as stroke, Alzheimer's, autism, and epilepsy. However, conventional neuroimaging modalities cannot easily be applied to mice, the most widely used model species for human brain disease studies. For instance, functional magnetic resonance imaging (fMRI) of mice requires a very high magnetic field to obtain a sufficient signal-to-noise ratio and spatial resolution. Functional connectivity mapping with optical intrinsic signal imaging (fcOIS) is an alternative method. Due to the diffusion of light in tissue, the spatial resolution of fcOIS is limited, and experiments have been performed using an exposed skull preparation. In this study, we show for the first time, the use of photoacoustic computed tomography (PACT) to noninvasively image resting-state functional connectivity in the mouse brain, with a large field of view and a high spatial resolution. Bilateral correlations were observed in eight regions, as well as several subregions. These findings agreed well with the Paxinos mouse brain atlas. This study showed that PACT is a promising, non-invasive modality for small-animal functional brain imaging.
\end{abstract}

Keywords: Functional Imaging, Photoacoustic Tomography, Electrical Stimulation.

\section{INTRODUCTION}

Conventional neuroimaging modalities cannot be easily translated to small animals. For instance, functional magnetic resonance imaging (fMRI) requires a very high magnetic field in order to obtain a sufficient signal to noise ratio (SNR) and spatial resolution for small animal imaging ${ }^{1}$. Functional connectivity mapping with optical intrinsic signal imaging (fcOIS) was recently introduced as an alternative method to image functional connectivity in mice ${ }^{2,3}$. In fcOIS, changes in local hemoglobin concentrations are determined based on changes in the reflected light intensity from the surface of the brain ${ }^{2,4}$. Therefore, neuronal activity can be measured through the neurovascular response, similar to the method used in fMRI. However, due to the diffusion of light in tissue, the spatial resolution of fcOIS is limited ${ }^{2,5}$, and the experiment has thus far been performed using an exposed skull preparation, which adds complexity for longitudinal imaging. Some other optical imaging methods such as optical coherence tomography may not have a sufficient penetration depth ${ }^{6}$. Photoacoustic computed tomography (PACT) is an emerging imaging technique that is based on the acoustic detection of optical absorption from tissue chromophores, such as oxy-hemoglobin $\left(\mathrm{HbO}_{2}\right)$ and deoxyhemoglobin $(\mathrm{Hb})^{7}$. This hybrid nature makes PACT capable of providing high resolution images of the brain while leaving the scalp intact ${ }^{8,9}$. And we expect that PACT can be developed into a powerful functional imaging modality for future brain research.

Photons Plus Ultrasound: Imaging and Sensing 2014, edited by Alexander A. Oraevsky, Lihong V. Wang, Proc. of SPIE Vol. 8943, 89432O - (c) 2014 SPIE · CCC code: 1605-7422/14/\$18 - doi: 10.1117/12.2036606 
In this paper, we utilize photoacoustic computed tomography imaging system to image the brain. The experiment was performed using a $532 \mathrm{~nm}$ wavelength laser. The photoacoustic signal was therefore directly proportional to the total hemoglobin concentration $(\mathrm{HbT})$.

\section{IMAGING SETUP}

A schematic of the photoacoustic computed tomography system is shown in Figure 1. A Nd:YAG laser (Quantel, Brillant B) was the excitation source, at a pulse duration of 4-6 ns and a pulse repetition rate of 10 $\mathrm{Hz}$. The laser beam was homogenized by an optical diffuser, resulting in a $2 \mathrm{~cm}$ diameter beam on the surface of the mouse's head. We monitored the laser power fluctuation using a photodetector, and the data was recorded into the computer. The data was then used in the reconstruction algorithm. The maximum light intensity at the surface was approximately $15 \mathrm{~mJ} / \mathrm{cm}^{2}$, below the ANSI limit of $20 \mathrm{~mJ} / \mathrm{cm}^{2}$ at $532 \mathrm{~nm}$ wavelength which is the isosbestic point ${ }^{10}$. The resulting photoacoustic signals were detected by a $5 \mathrm{~cm}$ diameter, full-ring ultrasonic transducer array (Imasonic Inc.) with 512 elements. The array had an 80\% bandwidth at a central frequency of $5 \mathrm{MHz}$. Within the $2 \mathrm{~cm}$ diameter field of view, the system had an axial resolution of $100 \mu \mathrm{m}$, a lateral resolution of 100-200 $\mu \mathrm{m}$, and an elevational resolution of $1.0 \mathrm{~mm}^{11}$. Atypical PACT image is shown in Figure 2.

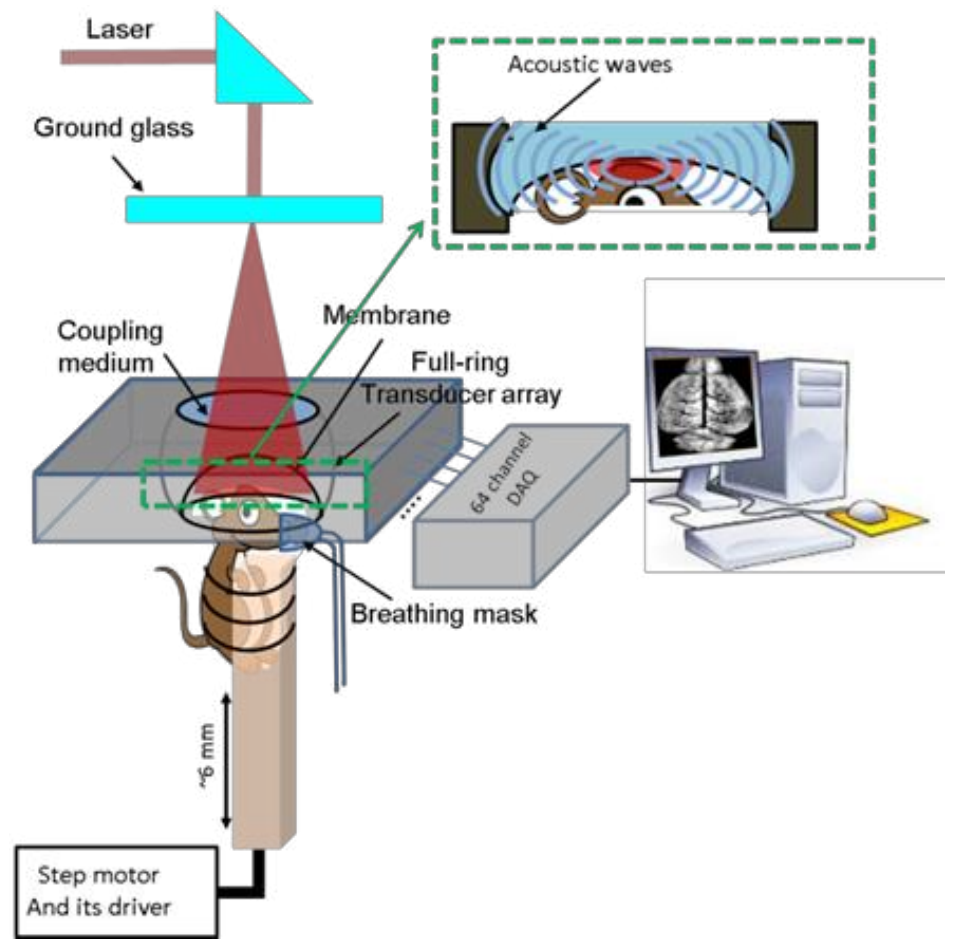

Figure 1. Schematic of the photoacoustic computed tomography (PACT) system

The photoacoustic signal was digitalized by a 64-channel data acquisition system, with a full-ring acquisition taking 1.6 seconds $^{8}$. For the experiments, we used 3-4 month old Swiss Webster mice. Before imaging, the animal was briefly anesthetized with $2 \%$ isoflurane, and the hair was removed with a hair remover lotion. The animal was then mounted on the imaging system. We changed the anesthesia from isoflurane to the mixture of ketamine and xylazine, because they provide stronger brain activity. $100 \mathrm{mg} / \mathrm{kg}$ ketamine and $10 \mathrm{mg} / \mathrm{kg}$ 
xylazine were mixed and administered intraperitoneally. For brain imaging, all experimental animal procedures were carried out in conformity with the guidelines of the US National Institutes of Health. The laboratory animal protocols for this work were in accordance with those approved by the Animal Studies Committee of Washington University in St. Louis.

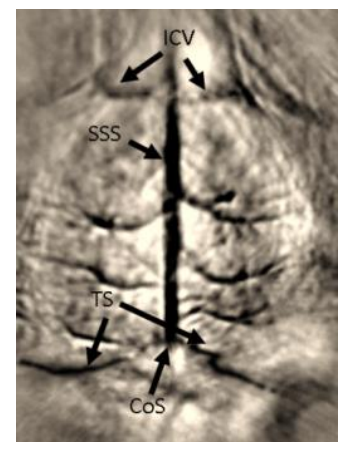

Figure2. Superficial cerebral vasculature image of a mouse brain. ICV: inferior cerebral vein, SSS: superior sagittal sinus, TS: transverse sinus, CoS: confluence of sinuses.

\section{ATLAS PREPARATION}

To support the findings obtained from this study, a horizontal view atlas of the functional regions of the mouse brain was reconstructed from coronal view plates of the Paxinos atlas, which are $120 \mu \mathrm{m}$ apart ${ }^{12}$. Since the elevational resolution of the PACT system is about $1 \mathrm{~mm}$, extensions of the coronal view plates from the surface of the cortex down to $1 \mathrm{~mm}$ deep were considered for reconstruction. Because the plates are projected into one horizontal plate, we call the reconstructed atlas the averaged horizontal atlas.

There were eight main regions reconstructed in the atlas. These regions include the olfactory bulb, limbic, parietal, somatosensory, retrosplenial, visual, motor, and temporal regions. Also several subregions within the main regions were reconstructed in the atlas.
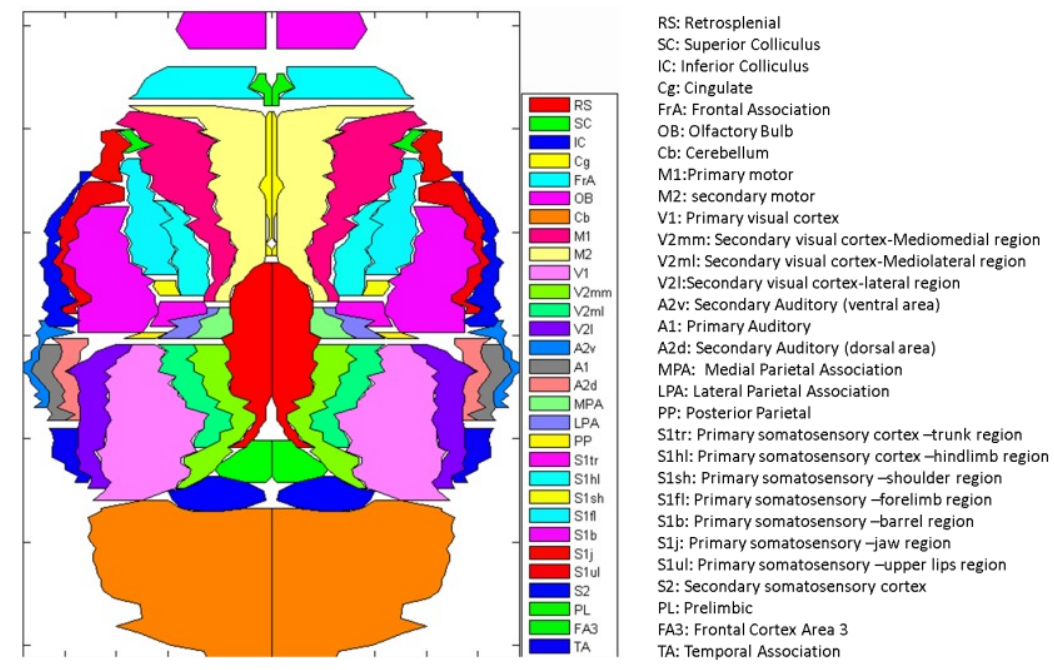

TA: Temporal Association

Figure 3. Functional regions from the Paxinos histological atlas. 


\section{RESULTS}

The fcPAT system was developed based on a 512-element full-ring ultrasonic transducer array (Figure 1), providing an in-plane resolution of $100 \mu \mathrm{m}$ [30]. Figure 2 shows an image of mouse cortical vasculature acquired noninvasively using this system. For better localization of functional regions, the photoacoustic images were co-registered to the Paxinos atlas using the landmarks shown in the vascular image (Figure 2). All mice used in this study were male ND4 Swiss Webster, anesthetized with ketamine/xylazine, and imaged for 10 minutes in resting state.

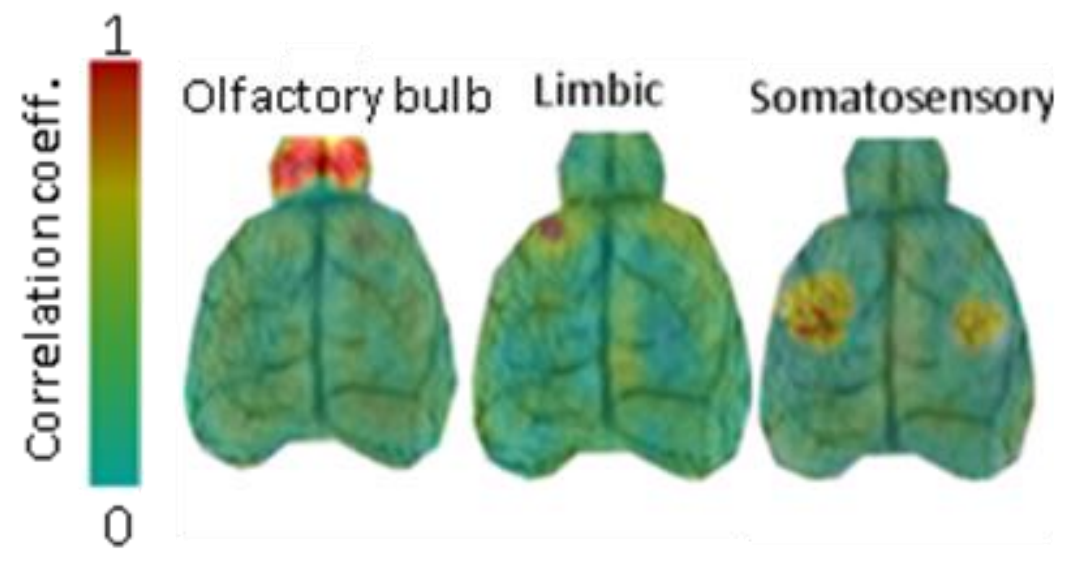

Figure 4. Functional connectivity maps in a live mouse brain acquired noninvasively by fcPAT. Correlation maps of Olfactory bulbs, Limbic, and Somatosensory regions.

In ${ }^{13}$, Fore- and hind-paw stimulation experiments were performed to confirm the locations of subregions in the somatosensory cortex ${ }^{14}$. Also, by subjecting the mouse to alternating normoxic and hypoxic conditions, strong and weak functional connectivities were observed, respectively.

\section{CONCLUSION}

In this study, we show for the first time, the use of photoacoustic computed tomography (PACT) to noninvasively image resting-state functional connectivity in the mouse brain, with a large field of view and a high spatial resolution. Bilateral correlations were observed in eight regions, and several subregions. This neuroimaging method can be used, in particular, for the patients under anesthesia, or for the patients who are not able to perform any cognitive task. We are planning to look into the resting state functional connectivity in the capillary level by using photoacoustic microscopy.

\section{ACKNOWLEDGEMENTS}

The authors acknowledge and thank Prof. James Ballard, for his close look at the manuscript. This work was sponsored in part by National Institutes of Health grants R01 EB000712, R01 EB008085, R01 CA134539, R01 CA159959, U54 CA136398, R01 EB010049, and DP1 EB016986 (NIH Director's Pioneer Award). L.W. has a financial interest in Microphotoacoustics, Inc. and Endra, Inc., which, however, did not support this work. 


\section{REFERENCES}

H. Benveniste and S. Blackband, "MR microscopy and high resolution small animal MRI: applications in neuroscience research," Progress in neurobiology 67 (5), 393-420 (2002).

E. Jonckers, J. Van Audekerke, G. De Visscher, A. Van der Linden, and M. Verhoye, "Functional connectivity fMRI of the rodent brain: comparison of functional connectivity networks in rat and mouse," PloS one 6 (4), e18876 (2011).

B.R. White, A.Q. Bauer, A.Z. Snyder, B.L. Schlaggar, J.M. Lee, and J.P. Culver, "Imaging of functional connectivity in the mouse brain," PloS one 6 (1), e16322 (2011).

A.W. Bero, A.Q. Bauer, F.R. Stewart, B.R. White, J.R. Cirrito, M.E. Raichle, J.P. Culver, and D.M. Holtzman, "Bidirectional relationship between functional connectivity and amyloid- $\beta$ deposition in mouse brain," The Journal of Neuroscience 32 (13), 4334-4340 (2012).

S. Bahar, M. Suh, M. Zhao, and T.H. Schwartz, "Intrinsic optical signal imaging of neocortical seizures: the'epileptic dip'," Neuroreport 17 (5), 499-503 (2006).

H. Niu, S. Khadka, F. Tian, Z.J. Lin, C. Lu, C. Zhu, and H. Liu, "Resting-state functional connectivity assessed with two diffuse optical tomographic systems," Journal of biomedical optics 16 (4), 046006-046006-046005 (2011).

Mohammad Avanaki, Adrian Gh Podoleanu, Mark C Price, Serena A Corr, and SA Hojjatoleslami, "Two applications of solid phantoms in performance assessment of optical coherence tomography systems," Applied optics 52 (29), 7054-7061 (2013).

Mohammadreza Nasiriavanaki, Adrian Gh Podoleanu, John B Schofield, Carole Jones, Manu Sira, Yan Liu, and Ali Hojjat, "Quantitative evaluation of scattering in optical coherence tomography skin images using the extended Huygens-Fresnel theorem," Applied optics 52 (8), 1574-1580 (2013).

L.V. Wang, "Tutorial on photoacoustic microscopy and computed tomography," Selected Topics in Quantum Electronics, IEEE Journal of 14 (1), 171-179 (2008).

J.M. Yang, C. Favazza, R. Chen, J. Yao, X. Cai, K. Maslov, Q. Zhou, K.K. Shung, and L.V. Wang, "Simultaneous functional photoacoustic and ultrasonic endoscopy of internal organs in vivo," Nature Medicine 18 (8), 1297-1302 (2012).

J. Gamelin, A. Maurudis, A. Aguirre, F. Huang, P. Guo, L.V. Wang, and Q. Zhu, "A real-time photoacoustic tomography system for small animals," Optics express 17 (13), 10489-10498 (2009).

2 Junjie Yao, Jun Xia, Konstantin I. Maslov, Mohammadreza Nasiriavanaki, Vassiliy Tsytsarev, Alexei V. Demchenko, and Lihong V. Wang, "Noninvasive photoacoustic computed tomography of mouse brain metabolism in vivo," Neuroimage $\mathbf{6 4}$ (0), 257-266 (2013).

A. Standard, "Z136. 1. American national standard for the safe use of lasers. American National Standards Institute," Inc., New York (1993).

J. Xia, Z. Guo, K. Maslov, A. Aguirre, Q. Zhu, C. Percival, and L.V. Wang, "Three-dimensional photoacoustic tomography based on the focal-line concept," Journal of biomedical optics 16 (9), 090505-090505-090503 (2011).

G. Paxinos and KBJ Franklin, "The mouse brain in stereotaxic coordinates: compact second edition," San Diego: Academic (2003).

Mohammadreza Nasiriavanaki, Jun Xia, Hanlin Wan, Adam Quentin Bauer, Joseph P Culver, and Lihong V Wang, "Highresolution photoacoustic tomography of resting-state functional connectivity in the mouse brain," Proceedings of the National Academy of Sciences 111 (1), 21-26 (2014).

Mohammadreza Nasiriavanaki, Jun Xia, and Lihong V. Wang. "High resolution functional photoacoustic computed tomography of the mouse brain during electrical stimulation." In SPIE BiOS, pp. 85813K-85813K. International Society for Optics and Photonics, (2013). 